\title{
A new scheme for initializing process-based ecosystem models by scaling soil carbon pools
}

S. Hashimoto ${ }^{\text {ab* }}$, M. Wattenbach ${ }^{\text {bc }}$, P. Smith ${ }^{\mathrm{b}}$

a Soil Resources Laboratory, Department of Forest Site Environment, Forestry and Forest Products Research Institute (FFPRI), Matsunosato 1, Tsukuba, Ibaraki 305-8687, Japan

b Institute of Biological and Environmental Sciences, School of Biological Sciences, University of Aberdeen, 23 St Machar Drive, Aberdeen, AB24 3UU, Scotland, UK

c Helmholtz Centre Potsdam, GFZ German Research Centre for Geosciences, Section 5.4 Hydrology, Telegrafenberg, 14473 Potsdam, GermanyCorrespondence to: S. Hashimoto

Soil Resources Laboratory, Department of Forest Site Environment, Forestry and Forest Products Research Institute (FFPRI), Incorporated administrative Agency, Matsunosato 1, Tsukuba, Ibaraki 305-8687, Japan, Tel: +81-29-829-8227, Fax: +81-29-874-3720, email: shojih@ffpri.affrc.go.jp 


\section{Abstract}

2 Process-based ecosystem models are useful tools, not only for understanding the forest carbon 3 cycle, but also for predicting future change. In order to apply a model to simulate a specific time 4 period, model initialization is required. In this study, we propose a new scheme of initialization 5 for forest ecosystem models, which we term a "slow-relaxation scheme", that entails scaling of 6 the soil carbon and nitrogen pools slowly during the spin-up period. The proposed slow-relation 7 scheme was tested with the CENTURY version 4 ecosystem model. Three different combinations 8 of scaled soil pools were also tested, and compared to the results from a fast-relaxation regime.

9 The fast-relaxation of soil pools produced unstable, transient model behaviour whereas 10 slow-relaxation overcame this instability. This approach holds promise for initializing ecosystem 11 models, and for starting simulations with more realistic initial conditions.

13 Keywords: ecosystem model, initialization, spin-up, soil carbon, forest

15 >We propose a new scheme of initialization for ecosystem models. > The scheme entails the 16 scaling of the soil pools slowly during the spin-up. > The slow-relaxation scheme was tested with 17 the CENTURY version 4 ecosystem model. > Slow-relaxation overcame the unstable model 18 behaviour seen using fast-relaxation. 


\section{Introduction}

Process-based ecosystem models are useful tools, not only for understanding the forest carbon cycle, but also for predicting future change. In general, ecosystem models consist of a number of conceptual pools, with flows between them, which combine to describe the ecosystem (Smith, 2001; Smith et al., 2002). Pools/processes included in such models include net primary productivity (NPP), biomass and soil carbon (e.g. Parton et al., 1988; Aber and Federer, 1992; Ito and Oikawa, 2002); in soil sub-models, soil decomposition process can be described by combining soil carbon/nitrogen pools (e.g. Jenkinson, 1990; Liski et al., 2005).

In order to apply a model for simulation of specific periods, initialization is required (e.g. Thornton and Rosenbloom, 2005). There are several methods for initialization: the first, and most commonly-used scheme, is to run the model until steady state, over for example a few thousand years, until the slower changing pools (e.g. soil organic carbon, SOC) cease to change. This initialization assumes equilibrium and as a result, net ecosystem production becomes zero. Using such an initialization scheme, it might happen that after the calibration of plant production modules, the estimated NPP agrees well with observed values, but the SOC is still over- or under-estimated. This can occur because of inaccuracies in process description in the models, or because the soil is not at equilibrium. Wutzler and Reichstein (2007), for example, showed that equilibrium soil carbon stock after the spin-up of Yasso model exceeded observed carbon stock by about $30 \%$ at a site that has not been disturbed for at least $150 \mathrm{yrs}$, in spite of the reasonable litter-fall input. The second method is either to use default pool distributions and sizes (Kirschbaum, 1999; Murty and McMurtrie, 2000), or to arbitrarily define the size of each initial pool. Using this method, outputs can exhibit unstable, or transient, behaviour when the model is run forward from the initial conditions, as the soil pools begin to receive carbon inputs from the plant production modules and change accordingly. Such unstable/transient behaviour does not represent a realistic response of the ecosystem to a sudden change of environment, but rather is an artefact caused by model flows to and from the pools not being in equilibrium. When using such initialization methods, model outputs for periods of transient behaviour artefacts are sometimes arbitrarily discarded, and the model output is used only after the model pools have stabilized. In 
most cases, it is almost impossible to obtain the actual values of all pools of models, especially when using models with many pools, and the fact is that most models are based on conceptual model pools that do not correspond well with measurable fractions (Smith et al., 2002; but see Zimmermann et al., 2007).

As described above, each scheme has its disadvantages, so alternative initialization options need to be found. Recently, several papers concerning model initialization have been published (e.g. Wutzler and Reichstein, 2007; Carvalhais et al., 2008). Carvalhais et al. (2008) proposed using a relaxed carbon cycle steady state assumption. They introduced a parameter that scales soil carbon pools in the Carnegie Ames Stanford Approach (CASA) model after spin-up: as mentioned above, soil carbon pools are at equilibrium after the normal spin-up. But at the end of the spin-up, soil carbon pools were decreased/increased by being multiplied by the scaling factor, which allowed the start of the simulation after spin-up with non-equilibrium soil carbon pools but with other biomass pools at equilibrium (Fig.1 (a)). Scaling soil carbon pools at the final stage of initialization for equilibrium decreased error of model NEP estimates, and enabled more realistic simulations of carbon budget and model parameterizations. In this study, we refer to this original scheme as a "fast-relaxation scheme".

In this paper we report the test of a fast-relaxation of steady state assumptions, as described by Carvalhais et al. (2008), with the CENTURY ver.4 model, and present an improved initialization scheme using slower relaxation of the steady state assumptions during initialization (Fig. 1 (b)), that enables a smoother transition from the spin-up run to the forward model run for models like CENTURY ver.4, which have feedbacks from soil nitrogen to plant growth and complex interactions between soil carbon and nitrogen pools.

\section{Materials and Methods}

The CENTURY ecosystem model ver. 4 was used. The CENTURY model can simulate carbon and nitrogen cycle in various ecosystems, from grassland to forest, and is one of the most widely-used plant-soil ecosystem models (Parton et al., 1988; Falloon and Smith, 2002). The 
model simulates plant production, plant biomass, soil carbon dynamics, soil nutrient cycles, and soil water and temperature. There are optional P and S routines which were not examined in this study.

We tested the initialization scheme at six sites in Japan from north to south using the mesh climate data 2000 (Japan Meteorological Agency). The database includes 30-year means for the period 1971-2000 of monthly climate data. We assumed the same mean monthly climate drivers for all years of the simulation. Here we report the result using the climate data at $33.7917^{\circ} \mathrm{N}$ and $133.6875{ }^{\circ} \mathrm{E}$ (mesh id: 50335555; average annual air temperature, $13.0^{\circ} \mathrm{C}$; average annual precipitation $2250 \mathrm{~mm})$.

The parameters used in this study were mostly from the default parameters in the AND parameter set (parameters tuned for coniferous forests in H. J. Andrews Experimental Forest), which was included in the CENTURY ver.4 package. The portions of sand, silt and clay were assumed to be 48 , 28, and $24 \%$, respectively. The ph was assumed to be 5.05 , and the bulk density was assumed to be $0.62 \mathrm{Mg} \mathrm{m}^{-3}$. Other parameters in the site file were the default parameters in tconif.100 of the CENTURY ver.4 files. For simplicity, no forest management was assumed in this study.

We show the results of both the fast-relaxation of steady state conditions used by Carvalhais et al. (2008), and a new initialization scheme (the slow-relaxation initialization) that we test for its ability to eliminate transient model behaviour after initialization. In the original application of the fast-relaxation scheme, the microbial and slow (intermediate) carbon pool in the CASA model was scaled at the end of the initialization spin-up period (Fig. 1(a)). In this study, a $2000 \mathrm{yr}$ spin-up interval was used; then, the scaling was done in the $1999^{\text {th }}$ year in the original initialization.

The CENTURY model has three main SOC pools, the active, slow and passive pools (Parton et al., 1987, 1988) that increase in turnover time from active through to passive, which we will refer to here as P1, P2 and P3, respectively. The soil carbon sub-model of CASA is based on the simplified structure of the CENTURY model and has three pools from decomposable pool (P1), 
102 Carvalhais et al. (2008) scaled P1 and P2; however, the decision of which pools to scale before equilibrium probably depends on the site conditions. In other words, at some sites, the decomposable SOC pool may not be at equilibrium, but the passive SOC pool or all SOC pools may not be at equilibrium at another site. This should be determined by careful examination of past land use, preliminary model simulations and comparison with observed data. We therefore tested three variants of scaling (Fig. 2): 1) scaling the two more decomposable pools (P1 and P2), 2) scaling the two least decomposable pools (P2 and P3), and 3) scaling all pools (all), and evaluated how the difference in pool scaling affects the soil carbon change. Also, the impact of increasing/decreasing the scaling of SOC on model output was examined. The amount of the SOC at equilibrium after a $2000 \mathrm{yr}$ spin-up was about $8000 \mathrm{gC} \mathrm{m}^{-2}$; then, the target level of SOC (i.e. the observed SOC) was set 1) to $6000 \mathrm{gC} \mathrm{m}^{-2}$, which represents a case where the observed SOC is smaller than the equilibrium SOC, and 2) to $10000 \mathrm{gC} \mathrm{m}^{-2}$, which represents a case where the observed SOC is higher than the equilibrium SOC. In short, we tested six combinations, three different combinations of which pools were scaled, and two differences between observed SOC and equilibrium SOC (larger and smaller than observed).The new slow-relaxation initialization scheme is conducted as described below. In order to fill pools in the model to some extent, the normal spin-up was conducted for the first $600 \mathrm{yrs}$, after which the target pools were controlled using the following procedure. The total spin-up interval was set to $2000 \mathrm{yrs}$ in this study; then the scaling was done from 600 to 1999 yr (Fig. 1 (b)). The detailed scaling protocol is as follows: during the initialization, the difference of soil organic carbon between model output and observed value (target level) was calculated, and the scaling factor $\eta$ was calculated at the end of the annual routine. $\eta$ was multiplied by the content of each organic carbon and nitrogen pool. In order to relax the scaling effect, we also introduce the "easing factor" $\alpha$, which was defined to reduce the effective difference between observed SOC and modelled SOC. When $\alpha$ is 1 , the gap between modelled SOC and observed SOC was adjusted at one scaling, while the gap was adjusted slowly when $\alpha$ is more than 1 . Please note that both organic carbon and nitrogen pools were scaled for both relaxations. For example, when scaling P1 and P2 pools, scaling was as follows: 
$130 \quad \eta=\frac{(P 1(t)+P 2(t))-\Delta / \alpha}{P 1(t)+P 2(t)}$

where $S O C_{\text {model }}$ is the modelled total SOC, $S O C_{\text {obs }}$ is the observed SOC (target level), $\triangle$ is the difference between modelled and observed SOC, $\eta$ is the scaling factor, $\alpha$ is the easing factor, and $t$ represents time step. P1N and P2N are the organic nitrogen pools corresponding to P1 and P2 carbon pools, respectively. In this study, $\alpha$ was set to be 1.0 for fast-relaxation, and 120.0 for slow-relaxation scaling. The large easing factor allows us to scale the modelled SOC slowly and then to reduce the gap between modelled SOC and observed SOC slowly. When scaling P2 and P3, $\eta$ was calculated using P2 and P3 and was multiplied to the P2 and P3 pools. When scaling all three pools (P1, P2, and P3), $\eta$ was calculated using total SOC (P1+P2+P3).

In short, in the fast-relaxation scheme, soil carbon and nitrogen pools are changed at one scaling (one model time step), whereas in the slow relaxation scheme those pools are adjusted through gradual scaling, controlled by the easing factor $\alpha$, and scaled over a longer period (600-1999).

\section{Results and Discussion}

The behaviours of the NPP and SOC during the spin-up (0-1999 yr) and normal simulation (2000-2100 yr) are shown in this study (Fig. 3). In all three schemes for scaling (P1+P2, P2+P3, all), the new slow-relaxation scheme showed stable simulation results. When using 
151 fast-relaxation, NPP showed unstable fluctuations after scaling (NPP around $2000 \mathrm{yr}$ in Fig. 3 152 (a1)(a2)(a3)), which is probably due to the feedback of soil nutrition to plant production and 153 complex soil C-N interactions in the CENTURY model. Using the slow-relaxation initialization scheme (Fig. 3 (b1)(b2)(b3)), there was no fluctuation, even after the end of the scaling period for the soil pool (2000 yr).

Three combinations of scaled pools were tested in this study. The difference between the scaled pools can be seen; the NPP and SOC in scaling when using "P2+P3" (Fig. 3 (b2)) and "all” pools (Fig. 3 (b3)) were very similar, but the result when scaling "P1+P2” (most decomposable; Fig. 3 (b1)) was different from those of the other two. In scaling "P2+P3" and "all” pools, the difference between NPP of higher SOC and that of lower SOC was less than $200 \mathrm{gC} \mathrm{m}^{-2}$ during the initialization, and was smaller than that of "P1+P2". The difference then reduced slowly after the end of the initialization phase (2000-2100 yr). The difference of SOC was also reduced after the end of the initialization, but the magnitude was very small (Fig. 3 (b2)(b3)). On the contrary, in scaling "P1+P2" (Fig. 3 (a1)), the difference in NPP was more than $300 \mathrm{gC} \mathrm{m}^{-2} \mathrm{yr}^{-1}$ during initialization and was reduced faster than those of scaling "P2+P3" and "all” after initialization. Accordingly, the difference between SOC was reduced more quickly than the difference when scaling “P2+P3” and “all”.

A similar phenomenon can be seen in fast-relaxation scaling (Fig. 3 (a1)(a2)(a3)); the model results from scaling "P2+P3" and "all” were very similar to each other, and the result from scaling "P1+P2" was different from the other two. The change in both NPP and SOC was large in scaling "P1+P2".

We tested the scheme at six sites in Japan from north to south, but only reported the results of a site where its climate is the average of Japan to keep the manuscript concise. Simulations at a southern site with much warmer climatic condition (average annual air temperature, $20.0^{\circ} \mathrm{C}$; not shown) showed that fast-relaxing scheme resulted in more unstable fluctuations after scaling, while the slow-relaxation scheme resulted in stable simulation results. However, in simulations at a northern site with much colder climatic condition (average annual air temperature, $3.5^{\circ} \mathrm{C}$ ), 
even the fast-relaxation scheme produced stability. We speculate that this could be related to the difference in speed of carbon and nitrogen dynamics: under warmer climate condition, carbon and nitrogen dynamics are faster than under colder climate condition (or a larger amount of carbon and nitrogen moves between pools at each time step); therefore, scaling, in particular fast scaling, has a larger impact on carbon and nitrogen dynamics within a model. Further tests of relaxation schemes under various conditions are needed to examine the stability and applicability of relaxation assumptions.

To our knowledge, the proposed method is the first scheme that can successfully scale both soil carbon and nitrogen pools. As recent studies suggest, nitrogen dynamics strongly constrain future terrestrial carbon dynamics (Zaehle et al., 2010). The proposed method will be useful for obtaining more realistic predictions of future carbon dynamics from simulations. We think it is important to further examine why the fast-relaxation yields unstable model behaviour. For example, although this is not tested, it may be possible that if the biomass pools were scaled with soil pools, even the fast-relaxation scheme might work well. Finally, it should be emphasized that appropriate information for initial conditions of carbon and nitrogen in plant and soil is of critical importance when the initialization scheme is applied, and that further comparison between results of observations and those of modelling will advance modelling approaches.

\section{Concluding remarks}

A new approach for initialization of process-based models, the slow-relaxation method of scaling soil carbon pools, is proposed in this study. Fast-relaxation scaling of soil carbon and nitrogen pools tended to result in unstable model behaviour in the CENTURY model; however, slow-relaxation scaling overcame such unstable model behaviour after initialization. Although further tests are needed, this approach holds promise for initializing ecosystem C-N models and for starting simulations with more realistic, and internally consistent, initial conditions. 
206 This work is supported in part by OECD Co-operative Research Programme on Biological 207 Resource Management for Sustainable Agriculture Systems and KAKENHI (18880032). PS is a 208 Royal Society-Wolfson Research Merit Award holder. The authors thank N. Carvalhais for 209 providing a supplementary material and explanation.

210

211 


\section{References}

213

Aber JD, Federer CA. A generalized, lumped-parameter model of photosynthesis, evapotranspiration and net primary production in temperate and boreal forest ecosystems. Oecologia 1992;92:463-474.

Carvalhais N, Reichstein M, Seixas J, Collatz GJ, Pereira JS, Berbigier P, Carrara A, Granier A, Montagnani L, Papale D, Rambal S, Sanz MJ, Valentini R. Implications of the carbon cycle steady state assumption for biogeochemical modeling performance and inverse parameter retrieval. Global Biogeochem Cycles 2008;22:GB2007 doi: 10.1029/2007GB003033.

Falloon P, Smith P. Simulating SOC changes in long-term experiments with RothC and CENTURY: model evaluation for a regional scale application. Soil Use Manag 2002;18:101-111.

Ito A, Oikawa T. A simulation model of the carbon cycle in land ecosystems (Sim-CYCLE): a description based on dry-matter production theory and plot-scale validation. Ecol Modell 2002;151:143-176.

Jenkinson DS. The turnover of organic carbon and nitrogen in soil. Philos Trans R Soc Lond B Biol Sci 1990;329:361-368.

Kirschbaum MUF. CenW, a forest growth model with linked carbon, energy, nutrient and water cycles. Ecol Modell 1999;118:17-59.

Liski J, Palosuo T, Peltoniemi M, Sievänen R. Carbon and decomposition model Yasso for forest soils. Ecol Modell 2005;189:168-182.

Murty D, McMurtrie RE. The decline of forest productivity as stands age: a model-based method for analysing causes for the decline. Ecol Modell 2000;134:185-205. 
Parton WJ, Schimel DS, Cole CV, Ojima DS. Analysis of factors controlling soil organic matter levels in great plains grasslands. Soil Sci Soc Am J 1987;51:1173-1179.

Parton WJ, Stewart JWB, Cole CV. Dynamics of C, N, P and S in grassland soils: a model. Biogeochemistry 1988;5:109-131.

Potter CS, Randerson JT, Field CB, Matson PA, Vitousek PM, Mooney HA, Klooster SA. Terrestrial ecosystem production: A process model based on global satellite and surface data. Global Biogeochem Cycles 1993;7:811-841.

Smith P, Soil organic matter modeling. In: Lal R, editor. Encyclopedia of Soil Science. New York: Marcel Dekker Inc; 2001. p. 917-924.

Smith JU, Smith P, Monaghan R, MacDonald J. When is a measured soil organic matter fraction equivalent to a model pool?. Eur J Soil Sci 2002;53:405-416.

Thornton PE, Rosenbloom NA. Ecosystem model spin-up: Estimating steady state conditions in a coupled terrestrial carbon and nitrogen cycle model. Ecol Modell 2005;189:25-48.

Wutzler T, Reichstein M. Soils apart from equilibrium - consequences for soil carbon balance modelling. Biogeosciences 2007;4:125-136.

Zaehle S, Friedlingstein P, Friend AD. Terrestrial nitrogen feedbacks may accelerate future climate change. Geophys Res Lett 2010;37:L01401 doi: 0.1029/2009GL041345.

Zimmermann M, Leifeld J, Schmidt MWI, Smith P, Fuhrer J. Measured soil organic matter fractions can be related to pools in the RothC model. Eur J Soil Sci 2007;58:658-667. 
257 Figure 1: Diagram showing the relaxation of the initial conditions of SOC showing (a) 258 fast-relaxation, scaling SOC at the end of initialization. (b) slow-relaxation, scaling SOC slowly, 259 which is proposed and tested in this study. Note that this example shows the case when observed 260 SOC was smaller than the equilibrium modelled SOC.

261 Figure 2: Three scalings tested in this study. Combinations of scaled pools differ.

262 Figure 3: NPP and SOC during and after the initialization; results of the original scaling method 263 (left column; a) and the slow relaxation method (right column; b). Upper columns (a1 and b1) are 264 results of scaling $\mathrm{P} 1+\mathrm{P} 2$, middle columns (a2 and b2) are results of scaling $\mathrm{P} 2+\mathrm{P} 3$, and lower 265 columns (a3 and b3) are results of scaling all pools. The solid line shows the result of the 266 increasing scaling (to $10000 \mathrm{gC} \mathrm{m}^{-2}, \eta>1$ ), and the broken line shows the results of the decreasing scaling (to $6000 \mathrm{gC} \mathrm{m}^{-2}, \eta<1$ ). Note that the time scale (x axis) is different before and after $2000 \mathrm{yr}$, to show the model behaviour clearly. 

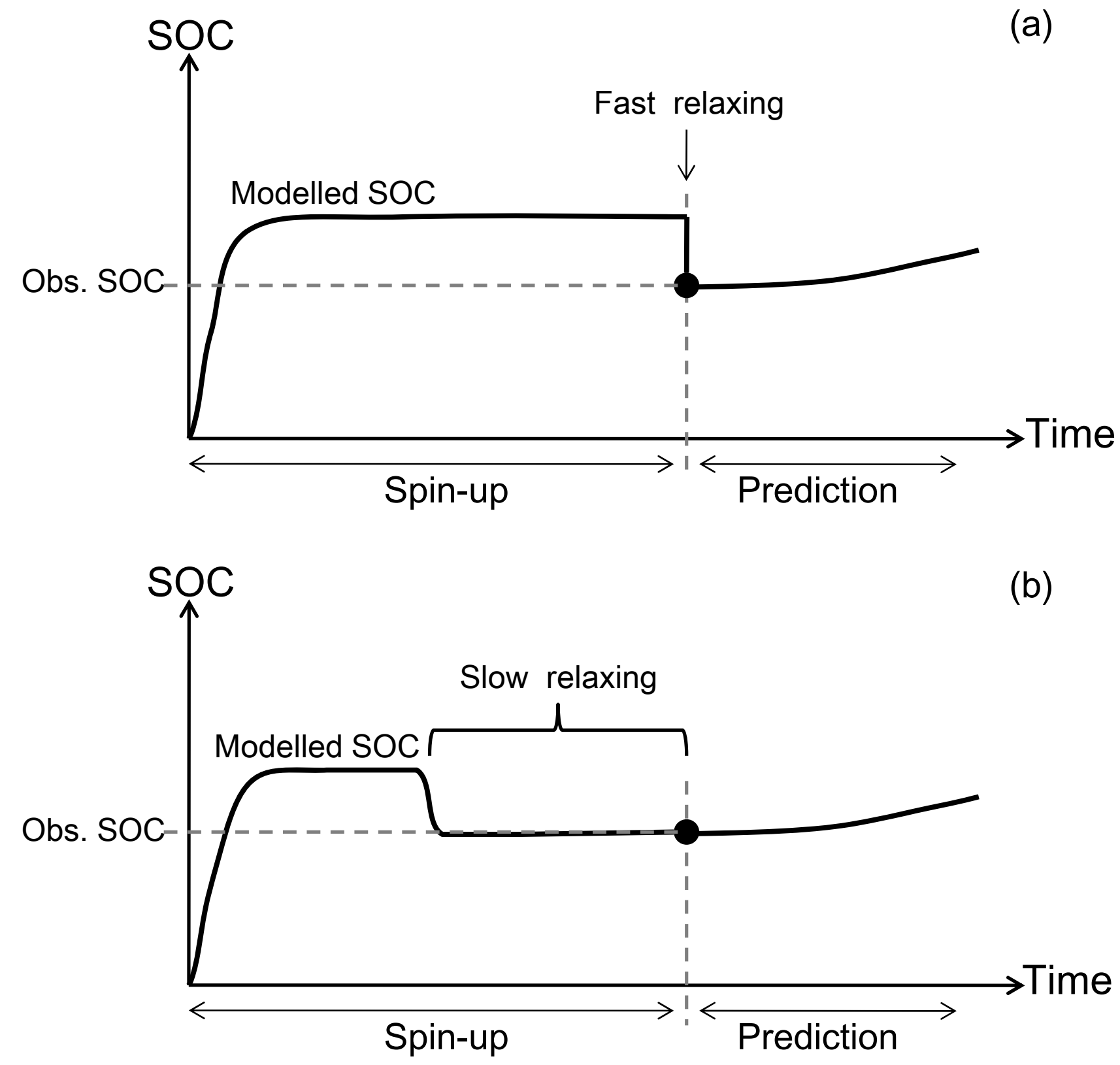


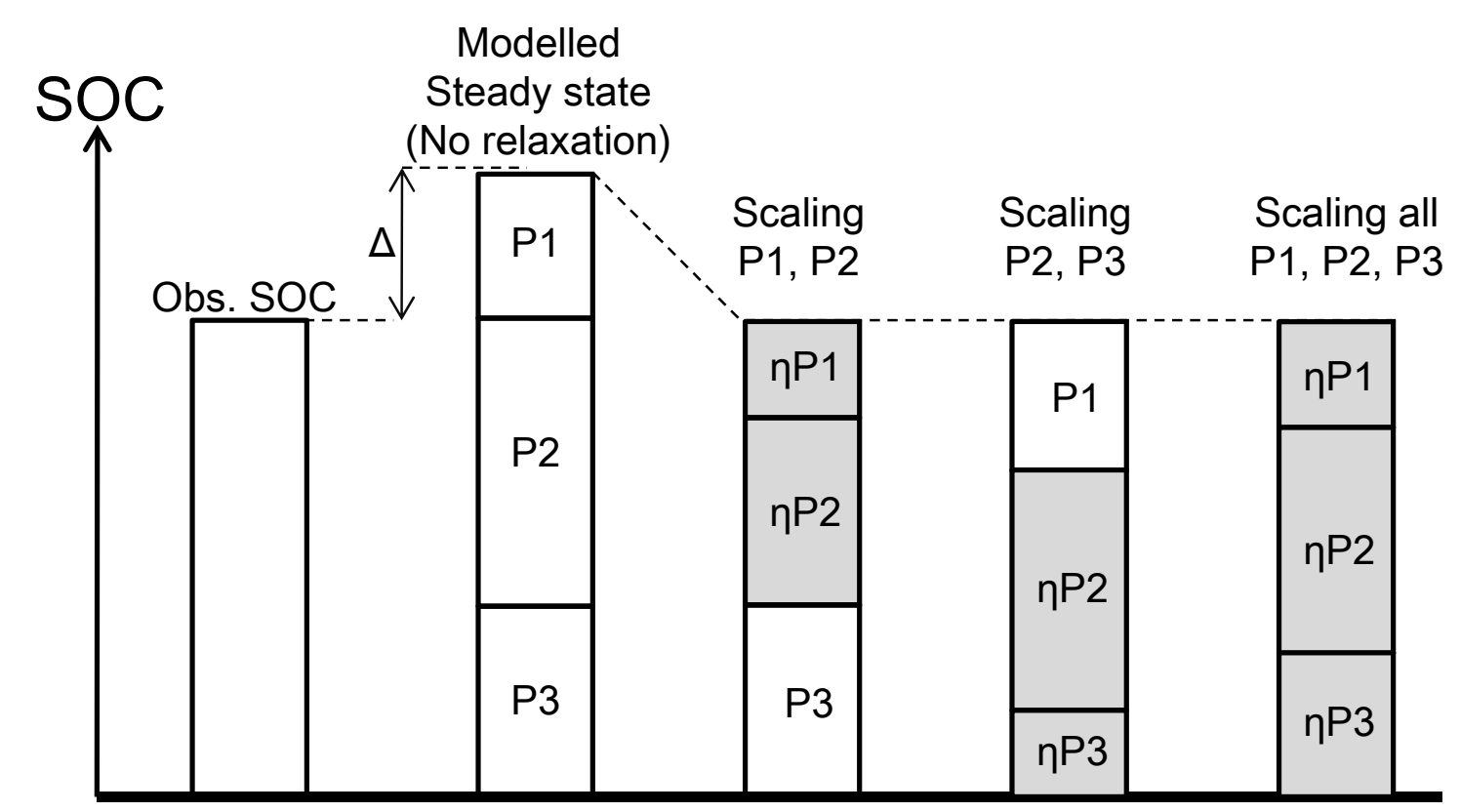


Fast relaxation
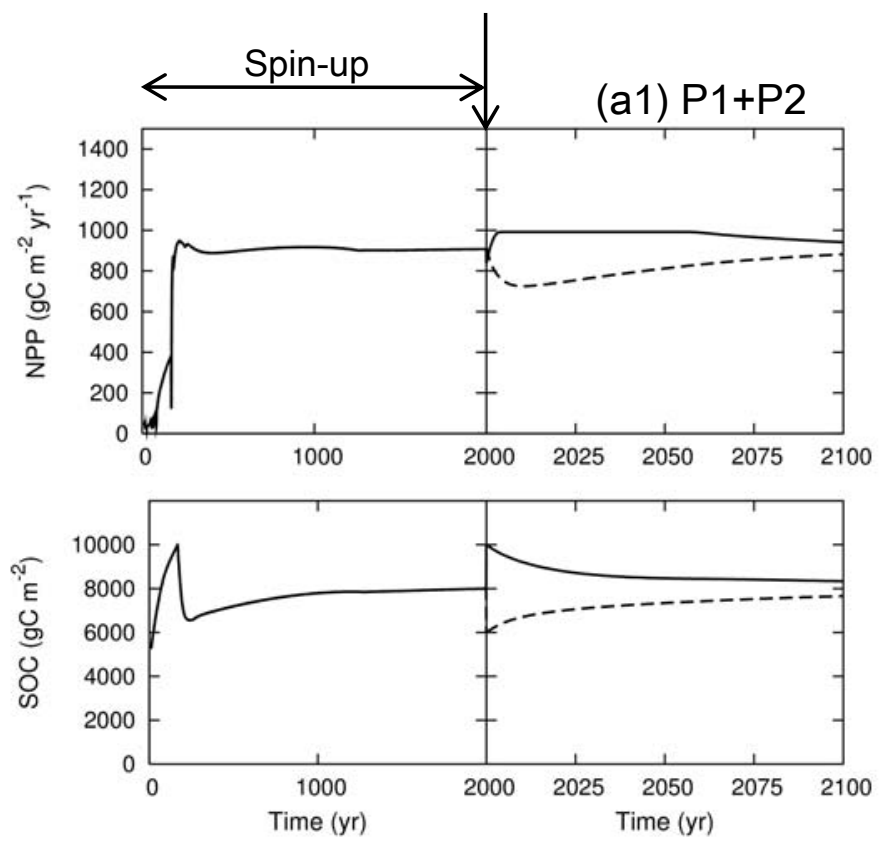

(a2) P2+P3
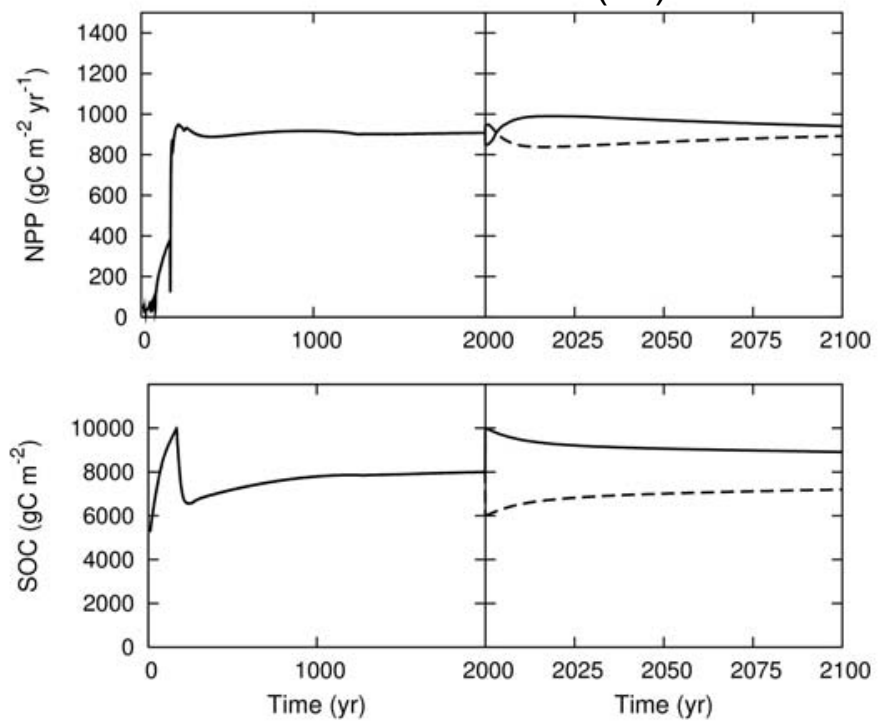

(a3) All
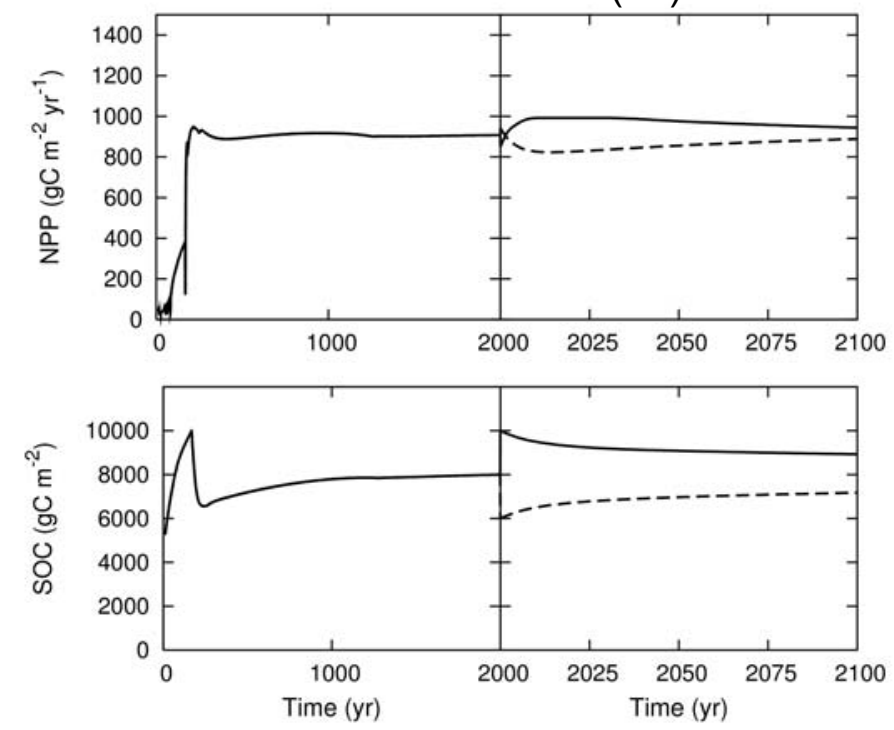
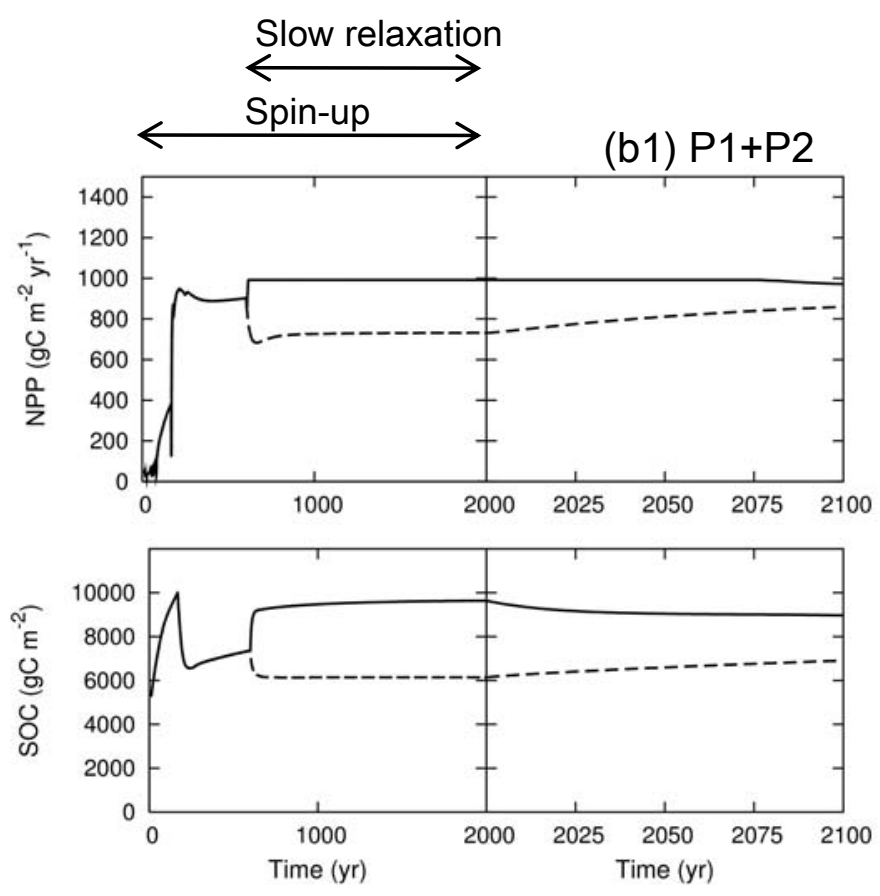

(b2) $\mathrm{P} 2+\mathrm{P} 3$
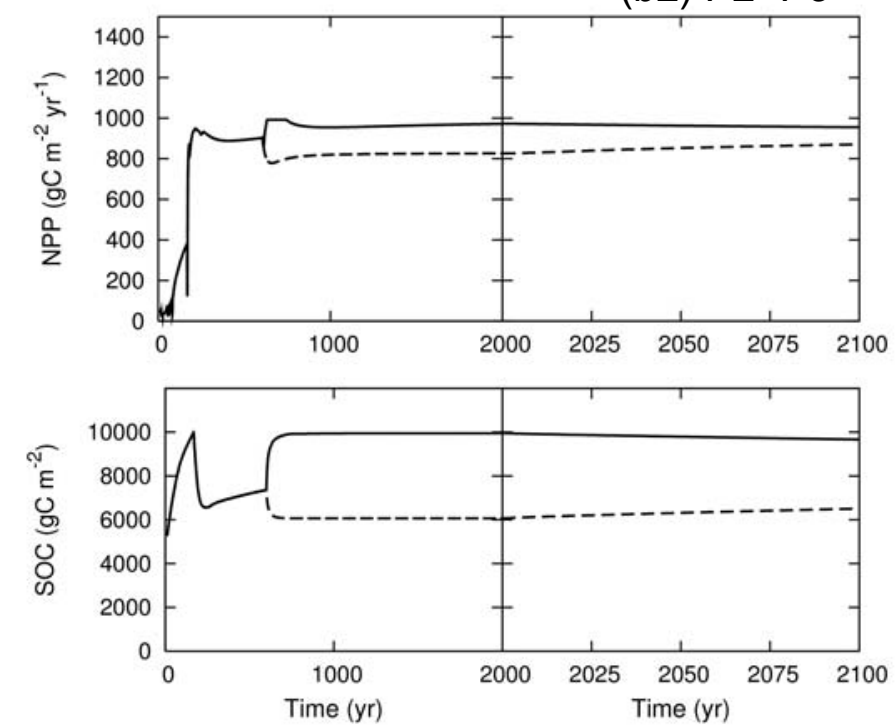

(b3) All
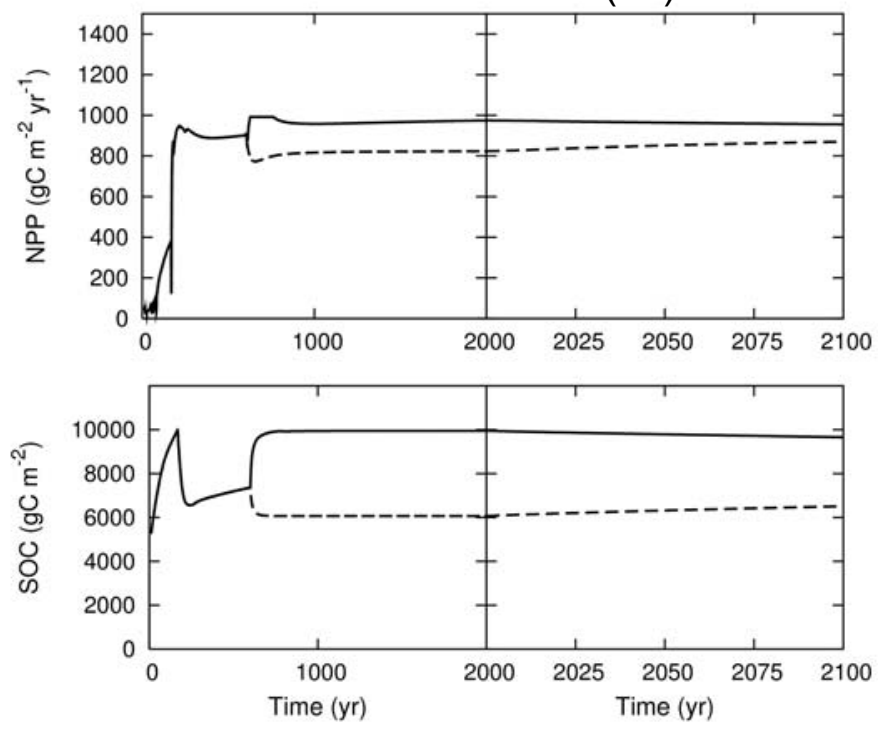